\title{
Inverter-Side Current Control of Grid-Connected Voltage Source Inverters With LCL Filter Based on Generalized Predictive Control
}

\author{
Marcos Gabriel Judewicz ${ }^{(}$, Member, IEEE, Sergio Alejandro González $^{\circledR}$, Member, IEEE, \\ Jonatan Roberto Fischer ${ }^{\circledR}$, Member, IEEE, Juan Francisco Martínez, \\ and Daniel Oscar Carrica, Senior Member, IEEE
}

\begin{abstract}
In this paper, an inverter-side current (ISC) control strategy for grid-connected voltage source inverter with LCL filters is proposed based on a generalized predictive control (GPC) formulation as it has the advantages of fixed switching frequency, a systematic design procedure and low computational complexity. As the GPC strategy is a subtype of continuous-control-set model predictive control strategies, a proper model for controlling the ISC is obtained, showing that a full-order model is preferred to its first-order counterpart. The controller is designed taking into consideration harmonic distortion limits and robustness against filter component variations. To this end, a complete three-wire LCL filter model is obtained and impedance sensitivity to each component variation is analyzed. Finally, its performance is evaluated by means of experimental results and compared with a predictive deadbeat controller.
\end{abstract}

Index Terms-Distributed power generation, electric current control, predictive control, pulse width modulation (PWM) inverters, three-phase electric power.

\section{INTRODUCTION}

$\mathbf{I}^{\mathrm{N}}$ N GRID-CONNECTED renewable energy sources, the grid-side voltage source inverter (VSI) injects distorted current into the grid, so its harmonic content must be controlled in order to meet power quality standards [1]. There are several sources of current harmonic distortion such as grid voltage distortion and power inverter nonlinearities (e.g., switching deadtimes), which can be mitigated by proper control techniques. In addition, the switching frequency of the power inverter adds a high-frequency ripple to the current waveform, which can be mitigated by the use of passive filters on the grid side. The most widely used is the L filter, but in order to achieve high attenuation at the frequency of interest, the inductance needed might be high and this leads to the

Manuscript received December 15, 2017; revised March 22, 2018; accepted April 1, 2018. Date of publication April 12, 2018; date of current version October 30, 2018. This work was supported in part by the Universidad Nacional de Mar del Plata under Grants ING494/17 and ING492/17 and in part by the Acencia Nacional de Promoción Científica y Tecnológica under Grant PICT-2014-1136. Recommended for publication by Associate Editor Hui Li. (Corresponding author: Marcos Gabriel Judewicz.)

The authors are with the Facultad de Ingeniería, Instituto de Investigaciones Científicas y Tecnológicas en Electrónica, Universidad Nacional de Mar del Plata, Mar del Plata 7600, Argentina, and also with the Consejo Nacional de Investigaciones Científicas y Técnicas, Buenos Aires C1033AAJ, Argentina (e-mail: marcosj@fi.mdp.edu.ar).

Color versions of one or more of the figures in this paper are available online at http://ieeexplore.iee.org.

Digital Object Identifier 10.1109/JESTPE.2018.2826365 construction of bulky and costly inductors. An alternative to this approach is the use of higher order passive filters, with smaller and cheaper components such as the LCL filter. Nevertheless, the inclusion of this type of filters presents some aspects to be considered. An LCL filter presents a resonant frequency, which can lead to oscillations unless some sort of passive or active damping systems are included. Passive damping is the simplest form, which needs the addition of a resistive element in series with the capacitor, at the expense of increased losses. On the other hand, active damping methods such as capacitor current feedforward (FF) loop make use of digital controllers, avoiding resistive losses. Nevertheless, they are prone to instabilities due to grid impedance variations [2].

In grid-connected VSIs the utilization of a current sensor is mandatory in order to protect the system against overcurrent situations. When using an LCL filter, this sensor is placed in the inverter-side inductor due to the fact that current dynamics is faster before filtering, and therefore a fast overcurrent protection can be implemented. This current sensor can also be used for current control, which leads to an inverter-side current (ISC) control loop. Closing the loop with the ISC measurement has shown to provide better stability margins than with the grid-side current (GSC) [3], but incurs in apparent power error, which can be mitigated by modifying the current reference phase shift subtracting the filter phase lag.

At present, there are several current control strategies for grid-connected VSI with LCL output filter, which can be classified into linear, nonlinear, and hybrid types. The nonlinear strategies include the sliding mode control method [4], finite control set model predictive control (FCS-MPC) [5], and deadbeat boundary control [6]. All these strategies drive the inverter switches directly, which can lead to variable switching frequency and a corresponding spread spectrum. This can lead to higher current total harmonic distortion $\left(\mathrm{THD}_{i}\right)$ and imposes a more stringent demand on output filter design. This can be mitigated by increasing the maximum switching frequency, although this measure also increases converter losses. In the case of FCS-MPC, a modification in the cost function can be used to reduce spectrum spreading [7], [8]. Another nonlinear approach is the use of recurrent neural networks [9], which make use of a trained neural network. However, it requires an iterative design process, which makes optimal performance a difficult goal. 
Linear control strategies include the proportional resonant (PR) control [10], pseudoderivative-feedback control [11], $H_{\infty}$ robust control [12], and state feedback [13] control. They make use of a pulsewidth modulator (PWM) in order to drive the inverter switches which guarantee a constant switching frequency. Their design makes use of a linear model of the system and seeks to achieve closed-loop stability in the frequency domain obtaining a suboptimal result. Another group of linear controllers are the so-called predictive strategies, which include predictive deadbeat (PDB) [14] and continuouscontrol-set model predictive control (CCS-MPC) [15]. They make use of the system model in order to obtain the future states and calculate the optimal control accordingly. In the case of deadbeat controllers, their sole objective is the maximization of current reference tracking dynamics. They have the disadvantage of high sensitivity to model mismatch, which can be mitigated by the inclusion of a Luenberger observer in its formulation [16]. On the other hand, CCS-MPC makes use of both a prediction and a control horizon, and can also handle system constraints by making an online optimization technique or by a predefined lookup table of control coefficients based on the system current state vector as in the CCS-MPC subtype known as Explicit MPC [15].

Online optimization in CCS-MPC strategies are useful in the presence of considerable amount of constraints, otherwise an offline optimization can be used such as another CCS-MPC subtype known as generalized predictive control (GPC). This strategy relies on a transfer function (TF) model of the system, including a filtered disturbance polynomial acting as a Luenberger observer, with its coefficients defining its gain, improving control system immunity to measurement noise. Also, it uses a quadratic cost function with error and control dynamics as input terms. This strategy has proven to meet current quality standards with low computational cost and model mismatch robustness in a VSI with L filter [17].

Therefore, in this paper, an ISC control strategy for gridconnected VSI with LCL filters is proposed based on a GPC formulation as it has the advantages of fixed switching frequency, a systematic design procedure and low computational complexity. As the GPC strategy is a subtype of CCS-MPC strategies, a proper model for controlling the ISC is obtained, showing that a full-order model is preferred to its first-order counterpart. The controller is designed taking into consideration harmonic distortion limits and robustness against filter component variations. To this end, a complete three-wire LCL filter model is obtained and impedance sensitivity to each component variation is analyzed. Finally, its performance is evaluated by means of experimental results and compared with a PDB controller.

The work is organized as follows: first, in Section II, a complete system model for the grid-connected VSI with LCL filter is obtained including possible component differences in each of the phases, following with its discrete-time formulation. Second, in Section III, GPC design considerations for ISC control are explained. Then, in Section IV, discretetime application details of the proposed GPC strategy are given, and in Section V, experimental results are shown.

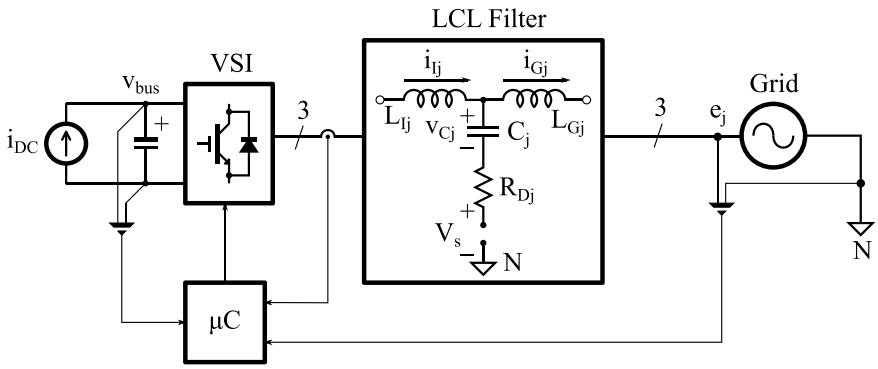

Fig. 1. LCL grid-connected VSI.

Finally, in Section VI, conclusions arising from this paper are discussed.

\section{SYSTEM MODEL}

The LCL grid-connected VSI is shown in Fig. 1. Input generator is modeled as a current source $i_{\mathrm{DC}}$ charging the DC bus at a voltage $v_{\text {bus }}$. A three-phase three-wire (3P3W) VSI configuration is used. The inverter switches use electronic drivers in order to generate the appropriate gate voltages and currents needed to change their state. The control system uses current and voltage measurements in order to generate the control action to be applied to the VSI. Grid voltage measurement is used to synchronize the sinusoidal current reference to the grid phase, usually by means of a phaselocked loop (PLL) algorithm, and can also be used to calculate a FF term in order to improve current control grid disturbance rejection capability. Bus voltage $v_{\text {bus }}$ is used to generate the PWM duty cycle $D$ as a function of control input $u$ as

$$
D=\frac{u}{v_{\text {bus }}}+0.5 \text {. }
$$

Output current $i_{G j}$ is injected to the grid through the LCL filter, which reduces the harmonic content generated by the inverter switching behavior. The LCL filter is composed of an inverter-side inductor $L_{I j}$, a capacitor $C_{j}$, a damping resistor $R_{D j}$, and a grid-side inductor $L_{G j}$. An equivalent loss resistance $r_{L j}$ can be included to account for inductor parasitic losses, but, for simplicity, it was not included in the control system design model. Finally, $e_{j}$ is the grid phase-to-neutral voltage. In all cases, $j=\{a, b, c\}$ represents the three different grid phases.

Despite the fact that a balanced model for the LCL filter is simpler to obtain, an unbalanced general model can be used to analyze the plant behavior in a more general case and will be used specifically to obtain an admittance sensitivity from component variations for control robustness analysis in a later section. The complete model of the LCL filter in a $3 \mathrm{P} 3 \mathrm{~W}$ configuration can be obtained by means of a state space formulation. First, the ISC $i_{I j}$ state equation is

$$
\frac{d i_{I j}(t)}{d t}=\frac{v_{j}(t)-i_{I j}(t) R_{D j}-v_{C j}(t)+i_{G j}(t) R_{D j}-V_{S}(t)}{L_{I j}}
$$

where $v_{j}$ is the inverter voltage applied to the load referred to neutral, $v_{C j}$ is the capacitor voltage, $i_{G j}$ is the GSC, and 
$V_{S}$ is the common node voltage of the LCL filter referred to neutral. Similarly, the $i_{G j}$ state equation is

$$
\frac{d i_{G j}(t)}{d t}=\frac{v_{C j}(t)+\left(i_{I j}(t)-i_{G j}(t)\right) R_{D j}-e_{j}(t)+V_{S}(t)}{L_{G j}} .
$$

Finally, the $v_{C j}$ state equation is

$$
\frac{d v_{C j}(t)}{d t}=\left(C_{j}\right)^{-1}\left[i_{I j}(t)-i_{G j}(t)\right] .
$$

As can be seen, the sum of the currents at the common node of the LCL filter must be zero, so

$$
\sum_{j}\left(i_{I j}-i_{G j}\right)=0
$$

and therefore

$$
\begin{gathered}
\sum_{j} i_{I j}=\sum_{j} i_{G j} \\
\sum_{j} \frac{d i_{I j}}{d t}=\sum_{j} \frac{d i_{G j}}{d t} .
\end{gathered}
$$

Replacing (2) and (3) in (6) results in

$$
\begin{aligned}
\sum_{j} & {\left[\frac{v_{j}(t)-i_{I j}(t) R_{D j}-v_{C j}(t)+i_{G j}(t) R_{D j}-V_{S}(t)}{L_{I j}}\right] } \\
= & \sum_{j}\left[\frac{v_{C j}(t)+\left(i_{I j}(t)-i_{G j}(t)\right) R_{D j}-e_{j}(t)+V_{S}(t)}{L_{G j}}\right] .
\end{aligned}
$$

Rearranging terms gives

$$
\begin{aligned}
V_{S}(t) \sum_{j}\left(\frac{1}{L_{I j}}+\frac{1}{L_{G j}}\right)= & \sum_{j} \frac{v_{j}(t)}{L_{I j}}+\left(i_{G j}(t)-i_{I j}(t)\right) R_{D j} \\
& \times\left(\frac{1}{L_{I j}}+\frac{1}{L_{G j}}\right)-v_{C j}(t) \\
& \times\left(\frac{1}{L_{I j}}+\frac{1}{L_{G j}}\right)+\frac{e_{j}(t)}{L_{G j}} .
\end{aligned}
$$

Defining

$$
\begin{aligned}
L_{p j} & =\left(\frac{1}{L_{I j}}+\frac{1}{L_{G j}}\right)^{-1} \\
L_{P} & =\left(\sum_{j} L_{p j}^{-1}\right)^{-1}
\end{aligned}
$$

and replacing in (8), $V_{S}(t)$ can be calculated as

$$
\begin{aligned}
V_{S}(t)=L_{P} \sum_{j} \frac{v_{j}(t)}{L_{I j}} & -i_{I j}(t) \frac{R_{D j}}{L_{p j}} \\
& -\frac{v_{C j}(t)}{L_{p j}}+i_{G j}(t) \frac{R_{D j}}{L_{p j}}+\frac{e_{j}(t)}{L_{G j}} .
\end{aligned}
$$

Replacing (9) both in (2) and (3) and using (4), the complete state space model of the LCL filter can be obtained. Considering a state space representation in the form

$$
\begin{aligned}
\dot{\mathbf{x}} & =\mathbf{A x}+\mathbf{B u} \\
y & =\mathbf{C} \mathbf{x}
\end{aligned}
$$

where

$$
\mathbf{x}=\left[\begin{array}{lllllllll}
i_{I a} & i_{G a} & v_{C a} & i_{I b} & i_{G b} & v_{C b} & i_{I c} & i_{G c} & v_{C c}
\end{array}\right]^{T}
$$

and

$$
\mathbf{u}=\left[\begin{array}{llllll}
v_{a} & e_{a} & v_{b} & e_{b} & v_{c} & e_{c}
\end{array}\right]
$$

and assuming a 3P3W LCL filter where component values per phase might be different, state matrices result in

$$
\begin{aligned}
\mathbf{A} & =\left[\begin{array}{lll}
\mathbf{A}_{a a} & \mathbf{A}_{a b} & \mathbf{A}_{a c} \\
\mathbf{A}_{b a} & \mathbf{A}_{b b} & \mathbf{A}_{b c} \\
\mathbf{A}_{c a} & \mathbf{A}_{c b} & \mathbf{A}_{c c}
\end{array}\right], \\
\mathbf{B} & =\left[\begin{array}{lll}
\mathbf{B}_{a a} & \mathbf{B}_{a b} & \mathbf{B}_{a c} \\
\mathbf{B}_{b a} & \mathbf{B}_{b b} & \mathbf{B}_{b c} \\
\mathbf{B}_{c a} & \mathbf{B}_{c b} & \mathbf{B}_{c c}
\end{array}\right], \\
\mathbf{C} & =\left[\begin{array}{lll}
1 & 0 & \ldots
\end{array}\right]
\end{aligned}
$$

where $\mathbf{C}$ is chosen so as to $y=i_{I a}$. When $j=k, k=\{a, b, c\}$

$$
\mathbf{A}_{j k}=\left[\begin{array}{ccc}
-R_{D j} \frac{K}{L_{I j}} & R_{D j} \frac{K}{L_{I j}} & -\frac{K}{L_{I j}} \\
R_{D j} \frac{K}{L_{G j}} & -R_{D j} \frac{K}{L_{G j}} & \frac{K}{L_{G j}} \\
\frac{1}{C_{j}} & -\frac{1}{C_{j}} & 0
\end{array}\right]
$$

with

$$
K=1-\frac{L_{P}}{L_{p j}}
$$

and

$$
\mathbf{B}_{j k}=\left[\begin{array}{cc}
L_{I j}^{-1}\left(1-\frac{L_{P}}{L_{I j}}\right) & -\frac{L_{P}}{L_{G j} L_{I j}} \\
\frac{L_{P}}{L_{G j} L_{I j}} & -L_{G_{j}}^{-1}\left(1-\frac{L_{P}}{L_{G_{j}}}\right) \\
0 & 0
\end{array}\right] .
$$

In this case, where $j \neq k$

$$
\begin{gathered}
\mathbf{A}_{j k}=\left[\begin{array}{ccc}
\frac{L_{P}}{L_{I k}} \frac{R_{D j}}{L_{p j}} & -\frac{L_{P}}{L_{I k}} \frac{L_{p j}}{R_{d j}} & \frac{L_{P}}{L_{p j} L_{I k}} \\
-\frac{L_{P}}{L_{G k}} \frac{R_{D j}}{L_{p j}} & \frac{L_{P}}{L_{G k}} \frac{L_{p j}}{L_{d j}} & -\frac{L_{P}}{L_{p j} L_{G k}} \\
0 & 0 & 0
\end{array}\right], \\
\mathbf{B}_{j k}=\left[\begin{array}{cc}
-\frac{L_{P}}{L_{I j} L_{I k}} & -\frac{L_{P}}{L_{G j} L_{I k}} \\
\frac{L_{P}}{L_{I j} L_{G k}} & \frac{L_{P}}{L_{G j} L_{G k}} \\
0 & 0
\end{array}\right] .
\end{gathered}
$$

From this state-space formulation, the TF matrix can be obtained by

$$
\mathbf{G}(s)=\mathbf{C}(s \mathbf{I}-\mathbf{A})^{-1} \mathbf{B}=\left[G_{1}(s) G_{2}(s) \ldots G_{6}(s)\right]
$$

where $\mathbf{I}$ is the identity square matrix the same size as the $A$ matrix. The TF used for controlling $i_{I a}$ with $v_{a}$, i.e., $i_{I a}(s) / v_{a}(s)$ is $G_{1}(s)$, therefore, $G_{p}(s)=G_{1}(s)$ is the plant TF to be used in the control system design procedure. Using the parameters shown in Table II, and assuming a balanced LCL filter, the resulting TF is

$$
G_{p}(s)=\frac{499.68}{s} \frac{s^{2}+840.9 s+5.606 \times 10^{7}}{s^{2}+1303 s+8.683 \times 10^{7}} .
$$

Equation (20) represents the admittance of the LCL filter which is composed of an integrator with gain and a pair of 
resonant zeroes and poles. The equivalent first-order model can be obtained from (20) as

$$
G_{p_{1 o}}(s)=\frac{k}{s}
$$

where $k=\lim _{s \rightarrow 0} s G_{p}(s)=322.61$ for the values given. Even though the first-order model is a good approximation of the LCL admittance for frequencies below the resonant frequency, using this model for the GPC design has certain differences which will be shown in a later section.

\section{A. Discrete-Time Model}

In order to use a discrete-time domain controller, the ISC needs to be sampled. This sampling process generates infinite copies of the original signal spectrum centered at the sampling frequency and its integer multiples. If the original signal is not band limited, the resulting measured spectrum suffers from aliasing, leading to a distorted measurement. This distortion affects the performance of PWM-based controllers for which an antialiasing (AA) filter inclusion is almost mandatory [14].

The simplest form for an AA filter is

$$
G_{f}(s)=\frac{1}{1+\frac{s}{s_{0}}}
$$

where $s_{0}=2 \pi f_{0}$ and $f_{0}$ is the $3-\mathrm{dB}$ bandwidth frequency. This type of filter should attenuate considerably the current ripple and add minimum phase shift at the control loop cutoff frequency. Therefore, the 3-dB bandwidth frequency should be a tradeoff between filtering and stability. In order to give the predictive control a more precise model of the system the AA filter TF is included in the plant model, resulting in

$$
G_{t}(s)=G_{p}(s) \times G_{f}(s)
$$

if using the complete model or

$$
G_{t}(s)=G_{p_{1 o}}(s) \times G_{f}(s)
$$

if using the first-order model. In order to use the system model in a discrete-time control system, the previous TF needs to be transformed to the discrete-time domain. As the control input from a PWM block to the plant can be seen as a staircase waveform, a zero-order hold discretization provides an exact match with respect to its continuous model [18]. Using a sampling period $T_{s}$, the resulting discrete-time TF is

$$
G_{t}\left(z^{-1}\right)=\frac{\sum_{i=2}^{M} b_{i} z^{-i}}{1+\sum_{i=1}^{N} a_{i} z^{-i}}=z^{-1} \frac{B\left(z^{-1}\right)}{A\left(z^{-1}\right)}
$$

where $M, N, b_{i}$, and $a_{i}$ depend on the LCL values. In the general unbalanced case and using the full-order model, $M=$ $N=6$; for the first-order approximation $M=3, N=2$. It is worth noting that numerator index starts at $i=2$, including an additional unit delay to the discrete TF, which represents the ISC control delay.

\section{ISC GPC DESIGN}

The GPC formulation is based on a controlled autoregressive and integrated moving-average transfer-function model, which has the form

$$
A\left(q^{-1}\right) \Delta y(k)=q^{-d} B\left(q^{-1}\right) \Delta u(k)+C\left(q^{-1}\right) v(k)
$$

where $q^{-1}$ is the unit delay operator in the discrete-time domain [18], $y(k)$ is the controlled variable, $u(k)$ is the controlled plant input, $v(k)$ is an unmeasurable disturbance signal, and $\Delta=1-q^{-1}$ is the discrete differentiation operator. Assuming that the coefficients of $A\left(z^{-1}\right)$ and $B\left(z^{-1}\right)$ are equal to the coefficients of $A\left(q^{-1}\right)$ and $B\left(q^{-1}\right)$, the obtained plant model can be used for the GPC design procedure. Also, $d$ is the number of additional discrete delays of the model, not included in $B\left(q^{-1}\right)$, which considering the model (25), results in $d=1$. Finally, $C\left(q^{-1}\right)$ is a disturbance model polynomial which sets the dominant eigenvalues of the GPC inherent Luenberger observer. In practice

$$
C\left(q^{-1}\right)=q^{-1}-0.8 q^{-2}
$$

which guarantees an optimal tradeoff between estimation speed of convergence and noise filtering [19].

Another design requirement for a GPC strategy regards setting its cost function parameters. The GPC cost function is

$$
\begin{aligned}
V(k)=\sum_{i=H_{w}}^{H_{p}}[\hat{y}(k+i \mid k)- & r(k+i)]^{2} \\
& +\sum_{i=0}^{H_{c}-1} \lambda[\Delta \hat{u}(k+i \mid k)]^{2}
\end{aligned}
$$

where $H_{w}$ is the initial prediction sample instant, $H_{p}$ is the prediction horizon length, $H_{c}$ is the control horizon length, and $\lambda$ is the control increment weighting factor. The argument $(k+i \mid k)$ means that the prediction of the variable at time $k+i$ is calculated at time $k$. Usually, $H_{w}=d+1$ to minimize computations, and $H_{c} \leq H_{p}$. The first term of the cost function weighs the error for each step over the prediction window in the optimization procedure and the second term weighs the rate of change of the control input over the control window. Each term weight relationship is set by $\lambda$ affecting controller behavior. In the absence of system constraints, the GPC optimization problem can be solved offline and is similar to an infinite-horizon linear quadratic regulator, assuming suffiently long $H_{p}-H_{w}$ and $H_{c}$ [20]. For the LCL application presented in this paper, $H_{w}=2, H_{p}-H_{w} \geq 32$, and $H_{c} \geq 30$. This has the advantage of reduced computational cost in comparison with online optimization algorithms.

After defining the model and the cost function parameters, the GPC controller is obtained by optimization through solving

$$
\frac{\partial V(k)}{\partial \Delta \hat{u}(k+i \mid k)}=0
$$

for $\Delta u(k)$ which results in

$$
\begin{aligned}
\Delta u_{\mathrm{opt}}(k) & =K_{\mathrm{GPC}} \mathcal{E}(k) \\
\mathcal{E}(k) & =T(k)-\Psi \hat{x}(k \mid k)-\Upsilon u(k-1),
\end{aligned}
$$



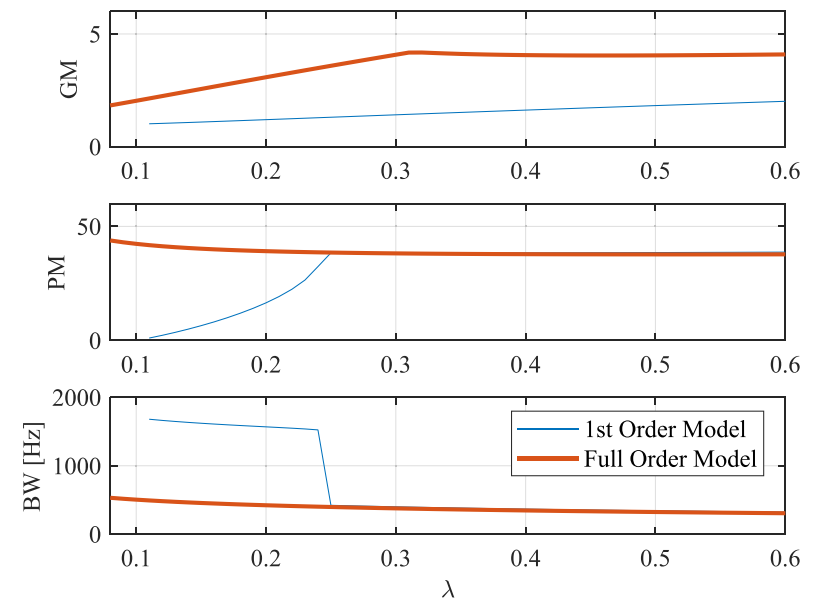

Fig. 2. Comparison of gain margin (top), phase margin (middle), and bandwidth (bottom) as a function of $\lambda$ between using a first-order or a fullorder model in GPC design.

where $K_{\mathrm{GPC}}$ is a vector of constant elements, $T(k)$ is the reference sequence, $\Psi \hat{x}(k \mid k)$ is the system free response up to $H_{p}$ based on the actual state estimation, and $\Upsilon u(k-1)$ is the system forced response up to $H_{c}-1$ based on the last control input applied. Then

$$
u_{\mathrm{opt}}(k)=u(k-1)+\Delta u_{\mathrm{opt}}(k) .
$$

For a complete analysis of GPC formulation [see [17]].

As previously stated, GPC requires a model in order to obtain the optimal control input as a function of previously applied inputs and measured outputs. Also, in an ISC control loop, the first-order model of (21) is valid for designing any control strategy. However, in the case of GPC, choosing the first-order model or the full-order model of (20) results in different control system behaviors, as shown in Fig. 2. The first value of $\lambda=0.08$ is the smallest value for which the full-order model-based design was stable which is also smaller than the value obtained using the first-order model, $\lambda=0.11$. In the $\lambda$ interval shown, gain margin is greater when using the full-order model than when using the first-order model. Also, for $0.08 \leq \lambda \leq 0.25$, using the full-order model results in much more phase margin than using the first-order model at the expense of control system bandwidth. As robustness is a desirable feature of any control system, using the full-order model for GPC design is a better choice than using its firstorder approximation. However, as can be seen in Fig. 2, for different values of $\lambda$, the controller behaves differently for a given model. Therefore, in order to design a GPC strategy, it is also necessary to find a value of $\lambda$ suitable for the current control of grid-connected VSIs. In [21], it is shown that several control structures can be obtained by the proper selection of both the prediction and control horizons, and $\lambda$. However, the effect of the precise value of this last parameter in the control system behavior is not analyzed. As shown in [17], the correct selection of $\lambda$ depends on the plant to be controlled, which is the approach also taken in this paper.

It is possible to design a GPC strategy-based solely on desired frequency-domain characteristics such as control bandwidth and phase margin. However, in practice, as with any predictive control strategy, the reliability of the control strategy design depends on the model accuracy. First of all, injected current into the grid needs to comply with quality standards in terms of $\mathrm{THD}_{i}$, which imposes a disturbance rejection capability of the controller to be used. Second, the controller needs to be robust against model mismatch, and be stable in a wide range of system variations. Both the disturbances and the model uncertainties are nondeterministic in nature, and therefore, its effect is difficult to be seen in a theoretical analysis. Consequently, both a current quality and robustness criteria for $\lambda$ selection are used and assessed by means of simulations and LCL admittance sensitivity to component variations, respectively.

\section{A. Current Quality Criterion}

One known source of current distortion in grid-connected VSIs is harmonic distortion $\left(\mathrm{THD}_{v}\right)$ present on grid voltages, which should be limited to a $5 \%$ at the point of common coupling if the grid operator complies with quality standards. Yet, another important source of distortion is the programmed dead time of the switching legs. Its purpose is to avoid short circuits between the dc-bus terminals due to the early turn on of one of the leg's switches while the other is still conducting. Nevertheless, it has a detrimental effect on injected current due to its nonlinear nature and represents a disturbance source which needs to be mitigated by the current controller [22]. Therefore, the controller not only should be capable of rejecting grid voltage harmonics but also higher order components added by the VSI itself.

In every linear current controller, the bandwidth-gain (BWG) product of the loop can be considered a figure of merit for its disturbance rejection capability: the higher the BWG the better its rejection capability. In the case of GPC, the value of $\lambda$ has a direct impact on BWG, i.e., lower values of $\lambda$ result in higher values of BWG. However, there is a lower bound on $\lambda$ for which smaller values make the system unstable. This is due to the fact that the optimal control law obtained from the optimization of the cost function does not guarantee system stability for every set of cost function parameters. As lower values of $\lambda$ result in higher BWG, they also result in lower phase and gain margins as the bandwidth approaches the sampling frequency. Therefore, in order to implement a stable controller, reject the dead time disturbance phenomenon and meet current quality standards, the value of $\lambda$ should stay between a lower and an upper bound. By means of simulation, the relationship between $\lambda$ and $\mathrm{THD}_{i}$ is shown in Fig. 3. The simulation was carried out using controller parameters of Table I and the LCL component values of Table II.

The lower value of $\lambda$ shown is the first at which the system is asymptotically stable, and results in the lowest $\mathrm{THD}_{i}$ achievable with this set of parameters. In addition, as can be seen, by increasing $\lambda$, current harmonic content progressively worsens, and for $\lambda>0.4$ approximately, the $\mathrm{THD}_{i}$ is never less than $5 \%$. Nevertheless, there are minima which are under the $5 \%$ limit of the standard but depend on the grid voltage harmonics' distribution which can be time variant. Therefore, 


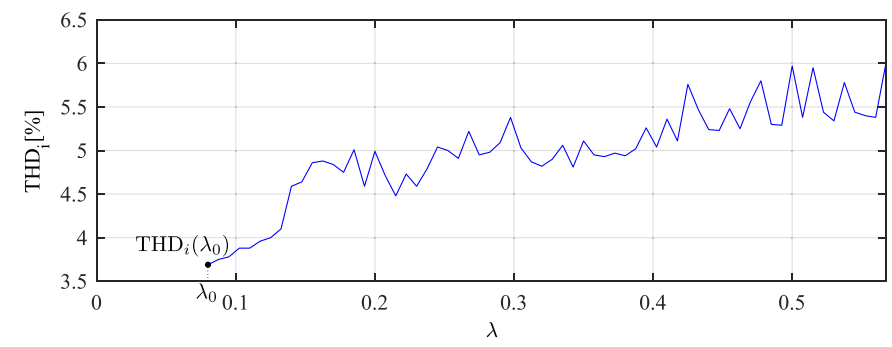

Fig. 3. Relationship between $\lambda$ and $\mathrm{THD}_{i}$ with $5 \%$ grid voltage distortion.

TABLE I

List OF CONTROL PARAMETERS

\begin{tabular}{|c|c|}
\hline Parameter & Value \\
\hline \hline Switching frequency $f_{s w}$ & $10 \mathrm{kHz}$ \\
\hline Sampling frequency $f_{s}$ & $30 \mathrm{kHz}$ \\
\hline FIR filter order $N$ & 3 \\
\hline Nominal RMS output current $I_{R M S}$ & $10 \mathrm{~A}$ \\
\hline AA filter 3-dB bandwidth frequency $f_{0}$ & $7 \mathrm{kHz}$ \\
\hline Programmed dead-time $T_{d}$ & $2.5 \mu \mathrm{s}$ \\
\hline Resistive losses $r_{L j}$ & $0.7 \Omega$ \\
\hline Grid RMS voltage $e_{j}$ & $220 \mathrm{~V}$ \\
\hline Grid voltage distortion $\mathrm{THD}_{v}$ & $5 \%$ \\
\hline
\end{tabular}

TABLE II

List of Nominal LCL FiLter COMPONEnt VALUeS

\begin{tabular}{|c|c|}
\hline Component & Value \\
\hline \hline$L_{I j}$ & $1.7 \mathrm{mH}$ \\
\hline$L_{G j}$ & $1.4 \mathrm{mH}$ \\
\hline$C_{j}$ & $15 \mu \mathrm{F}$ \\
\hline$R_{D j}$ & $1 \Omega$ \\
\hline
\end{tabular}

choosing $\lambda<0.15$ guarantees compliance even under distorted grid conditions.

\section{B. Robustness Criterion}

The following analysis will be useful for determining the most critical parameter variation for the GPC design robustness criteria. In order to carry on this analysis, the LCL nominal component values were chosen from a $10 \mathrm{~kW}$ gridconnected VSI application, and shown in Table II.

The procedure to select these values was taken from [23] and are identical at each phase. The upcoming analysis takes into consideration same-phase and different-phase finite parameter variations, and the percentual admittance magnitude $S(|Y|)[\%]$, the admittance phase shift $S(\angle Y)$ sensitivities and the resonant frequency shift $S\left(f_{r}\right)$ sensitivities to this variations were chosen as the criteria for determining which of the components' variation is the most critical for robustness, and can be defined as

$$
\begin{aligned}
S(|Y|)[\%] & =\frac{|d| Y(f)|/ d \gamma|}{|Y(f)|} \times 100 \\
S(\angle Y) & =|d(\angle Y(f)) / d \gamma| \\
S\left(f_{r}\right) & =\left|d f_{r} / d \gamma\right|
\end{aligned}
$$

TABLE III

Resonance FreQuency Shift SENSITIVITy to SAME-PHASE COMPONENT VARIATION

\begin{tabular}{|c|c||c|c|}
\hline Component & $S\left(f_{r}\right)$ & Component & $S\left(f_{r}\right)$ \\
\hline \hline$L_{I a}$ & $-1.8 \mathrm{~Hz}$ & $L_{I b}$ & $-0.8 \mathrm{~Hz}$ \\
\hline$L_{G a}$ & $-7.9 \mathrm{~Hz}$ & $L_{G b}$ & $-0.9 \mathrm{~Hz}$ \\
\hline$C_{a}$ & $-8.8 \mathrm{~Hz}$ & $C_{b}$ & $-2.1 \mathrm{~Hz}$ \\
\hline$R_{D a}$ & $0.1 \mathrm{~Hz}$ & $R_{D b}$ & $0.0 \mathrm{~Hz}$ \\
\hline
\end{tabular}

where $Y$ is the admittance of the LCL filter as seen from one of the phases, $|Y(f)|$ is the admittance magnitude as function of frequency, $\angle Y(f)$ is the admittance phase shift as function of frequency, $f_{r}$ is the resonant frequency, and $\gamma=L_{I x}, L_{G x}, C_{x}, R_{D x}$ with $x=a, b, c$ is the variable LCL component for which the sensitivity is calculated.

In order to determine which parameter variation has the worst effect on the control system, the maximum control bandwidth (BW) must be known. In the case of GPC, the maximum BW is achieved with the minimum $\lambda$ as can be seen in Fig. 2, which is between 500 and $550 \mathrm{~Hz}$. A total of eight components can affect the admittance seen from any phase, four samephase components and four different-phase components taken from any of the other phases.

1) Same-Phase Finite Parameter Variations: This type of variations occur at the same phase of the controlled ISC, i.e., if controlling $i_{I a}$, then the components which vary can be $L_{I a}$, $C_{a}, R_{D a}$, or $L_{G a}$. The percentual admittance magnitude sensitivity to these variations as a function of frequency is shown in Fig. 4(a). As can be seen, both $L_{I a}$ and $L_{G a}$ variations have a greater effect than $C_{a}$ and $R_{D a}$ at the frequencies inside the control bandwidth. A different situation occurs for the admittance phase shift sensitivity shown in Fig. 4(b). Here, the phase shift sensitivity is greater for $C_{a}$ up to $80 \mathrm{~Hz}$ overlapping with $L_{G a}$ from there on. Finally, in Table III, resonant frequency shift sensitivity is shown for each of the same-phase elements in the two leftmost columns, where $C_{a}$ variations show the greatest resonant frequency shift effect.

2) Different-Phase Finite Parameter Variations: This type of variations occur at a different phase of the controlled ISC, i.e., if controlling $i_{I a}$, then the components which vary can be $L_{I b}, C_{b}, R_{D b}$, or $L_{G b}$, for example. The variation of the $c$ phase components has the same effect than the variation of the $b$ phase components on the $a$ phase admittance. Therefore, it is only necessary to analyze the effect of the component variation on one of the $b$ or $c$ phases only. The percentual admittance magnitude sensitivity to these variations as a function of frequency is shown in Fig. 5(a) and the admittance phase shift sensitivity is shown in Fig. 5(b). As can be seen, $C_{b}$ variations have the greatest effect in both parameters at the frequencies inside the control bandwidth. Finally, in Table III, resonant frequency shift sensitivity is shown for each of different-phase elements in the two rightmost columns, where $C_{b}$ variations show the greatest resonant frequency shift effect.

As can be seen, the LCL filter admittance magnitude has an overall greater sensitivity from the same-phase parameter variation than from different-phase variations. Within the 


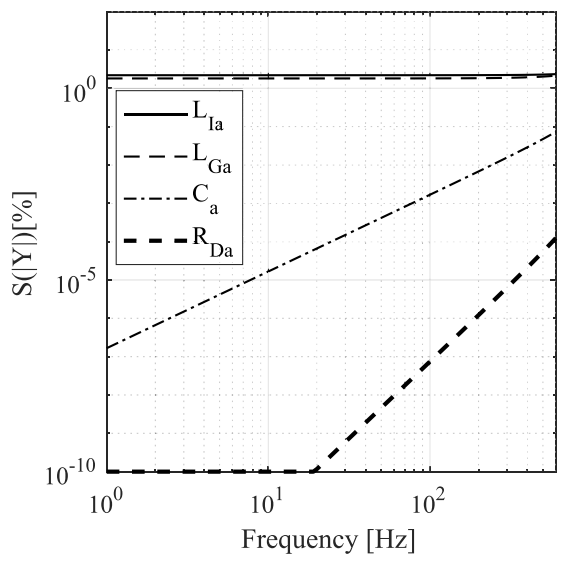

(a)

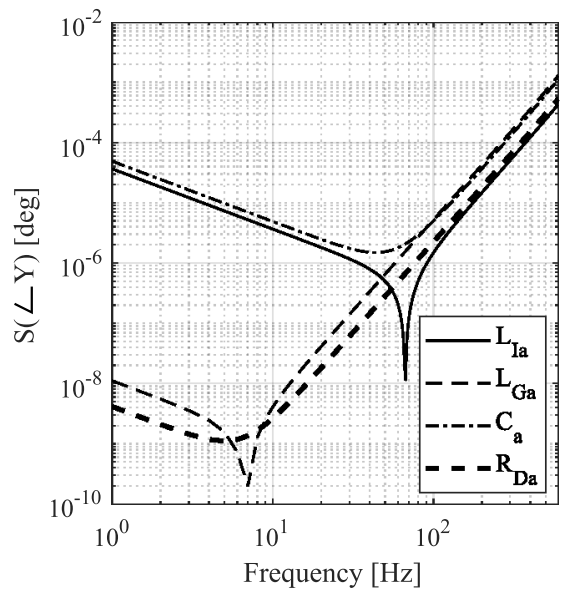

(b)

Fig. 4. (a) Same-phase percentual admittance magnitude sensitivity $S(Y)$ as a percentage of nominal admittance for $1 \leq f \leq 600 \mathrm{~Hz}$. (b) Admittance phase shift sensitivity $S(\angle Y)$ for $1 \leq f \leq 600 \mathrm{~Hz}$.

same-phase parameter variations, both the inverter-side and grid-side inductance have the greatest effect on admittance magnitude. In this case, as the inverter-side inductance has a greater inductance value than the grid-side inductance its variation contributes more to admittance magnitude variations than the grid-side inductance.

On the other hand, the admittance phase shift is primarily affected near the control bandwidth almost equally by $C_{a}$ and $L_{G a}$ and at low frequency by $C_{b}$ or $C_{c}$. However, the amount of phase shift can be considered a minor effect in comparison with the gain variations shown.

Finally, resonant frequency shift is larger for the same-phase components, with $C_{a}$ and $L_{G a}$ variations having the largest effect of all. However, in order for the resonant frequency to be near the control bandwidth, $\Delta C>300 \%$ for all capacitors or $\Delta L_{G a}>400 \%$ which are very difficult situations to happen in practice.

From the previous analysis, the admittance gain variations are taken as the most possible cause of instability for GPC strategies in grid-connected VSIs with LCL filters, with $L_{I a}$ and $L_{G a}$ having the most critical effect in its variation. However, for $L_{G a}$ variations between $\pm 100 \%$ of its nominal

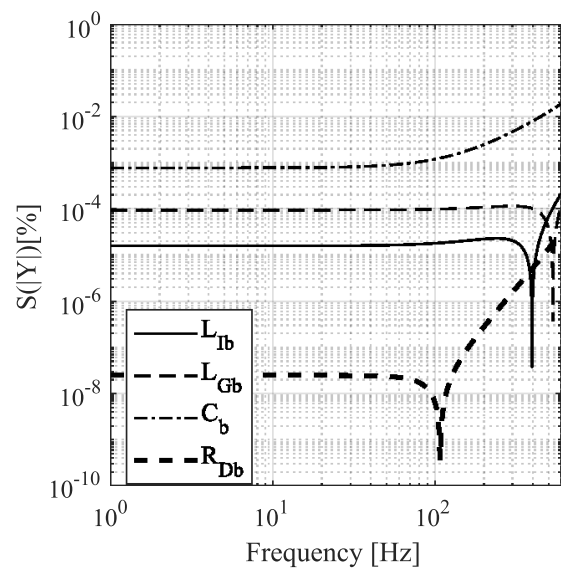

(a)

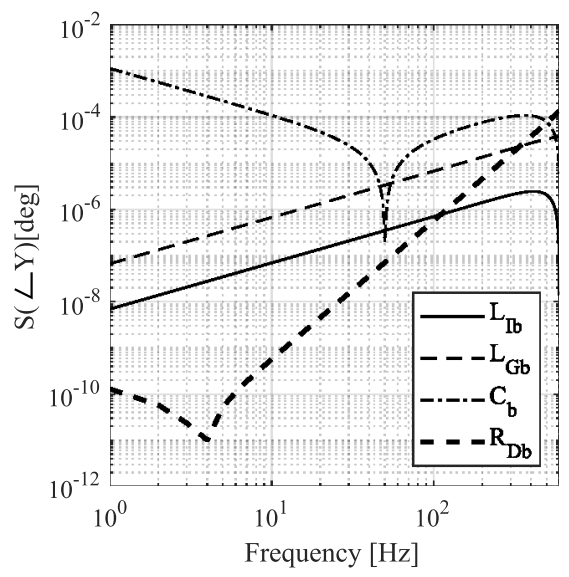

(b)

Fig. 5. (a) Different-phase percentual admittance magnitude sensitivity $S(Y)$ as a percentage of nominal admittance for $1 \leq f \leq 600 \mathrm{~Hz}$. (b) Admittance phase shift sensitivity $S(\angle Y)$ for $1 \leq f \leq 600 \mathrm{~Hz}$.

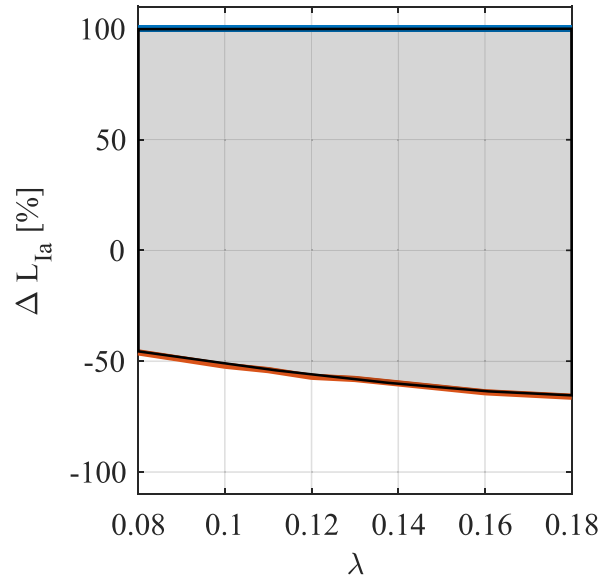

Fig. 6. Relationship between $\lambda$ and maximum allowable parameter variation of $L_{I m}$ with respect to the real inductance $L_{I r}$.

value, GPC remains stable for $0.08 \geq \lambda \geq 0.18$. Instead, for $L_{I a}$ variations of the same kind, stability limits are shown in Fig. 6, where the filled zone is the stable operation and it can be seen that for inductance increments up to $100 \%$ the GPC strategy remains stable but for reductions, its allowable 
TABLE IV

LIST OF GPC TF COEFFICIENTS

\begin{tabular}{|c|c||c|c|}
\hline Coefficient & Value & Coefficient & Value \\
\hline \hline$b_{0}$ & 14.85 & $a_{1}$ & -1.169 \\
\hline$b_{1}$ & -32.13 & $a_{2}$ & -0.1264 \\
\hline$b_{2}$ & 30.49 & $a_{3}$ & 0.6606 \\
\hline$b_{3}$ & -12.41 & $a_{4}$ & -0.2378 \\
\hline$b_{4}$ & 0.148 & $a_{5}$ & -0.1274 \\
\hline
\end{tabular}

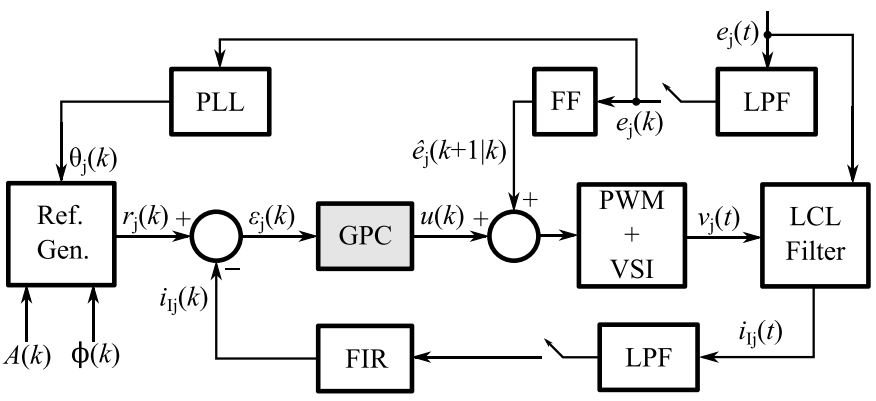

Fig. 7. Control system block diagram.

amount depends on the selection of $\lambda$. It is shown that for increasing values of $\lambda$ the control system can deal with greater parameter variations. In order to obtain the most robust design, and simultaneously, comply with current quality standards, choosing $\lambda=0.15$ is the right decision with an expected $\mathrm{THD}_{i}<5 \%$ under a highly distorted grid $\left(\mathrm{THD}_{v}=5 \%\right)$ and stable operation for $L_{I a_{n o m}}+100 \%-62 \%$. With this selection, the resulting GPC TF can be obtained following the procedure given in [17] as:

$$
G_{\mathrm{GPC}}\left(z^{-1}\right)=\frac{\sum_{i=0}^{4} b_{i} z^{-i}}{1+\sum_{i=1}^{5} a_{i} z^{-i}}
$$

which is the TF between the current error and the control input applied to the VSI, with the coefficients shown in Table IV.

\section{IMPLEMENTATION OF ISC GPC}

The control system block diagram is shown in Fig. 7. As can be seen, each of the sensed variables is passed through a lowpass filter such as the ones described in Section II-A whose pole frequency $f_{0}$ can be set near the switching frequency $f_{\text {sw }}$ by means of oversampling techniques.

Oversampling techniques allow the inclusion of a digital filter in order to reduce the switching frequency components in the current measurement waveforms used in the control system. This is achieved by setting the sampling frequency $f_{s}$ as an integer multiple of the switching frequency, i.e., $f_{s}=$ $N \times f_{\text {sw }}$. A moving average finite-impulse response (FIR) digital filter of the form

$$
\bar{x}(k)=\frac{1}{N} \sum_{i=-\frac{N-1}{2}}^{\frac{N-1}{2}} x(k+i)
$$

can be used, where $N$ is an odd integer number of samples, in order to have the same forward and backward samples of the moving average window. As can be seen, the filter is noncausal so a modification is needed in order to make possible its realization. For $N=3$, the modified FIR filter has the form in the $z$-domain [17]

$$
H_{\text {FIR }}\left(z^{-1}\right)=\frac{2}{3}+\frac{1}{3} z^{-1}+\frac{1}{3} z^{-2}-\frac{1}{3} z^{-3}
$$

where a linear extrapolation was used in order to make an estimation of the current sample. For $N>3$, the linear extrapolation is not useful and more complex techniques should be used in order to obtain the forward samples estimation.

Using the FIR filter generates a notch at the switching frequency which results in a greater attenuation than using only the AA filter alone. Besides, even if it adds phase shift at the notch frequency, the control cutoff frequency is at least a decade lower keeping phase margin unaltered. The filtered variables are sampled by means of a sample and hold $(S \& H)$ circuit and applying an oversampling strategy with $f_{s}=30 \mathrm{kHz}$ allows that $f_{0}=7 \mathrm{kHz}$ without aliasing.

In the case of the grid voltage measurements $e_{j}(k)$, they are used by a software-based PLL with variable sampling period in order to reject grid frequency variations [24], and thus obtaining the instantaneous phase values $\theta_{j}(k)$ with $j=a, b, c$ for each $3 \mathrm{P}$ grid component. They are also used by a FF block in order to improve control system grid disturbance rejection capability at the ISC. The FF calculation is carried out by means of a linear extrapolation [25] of the form

$$
\hat{e}_{j}(k+1 \mid k)=2.5 e_{j}(k)-1.5 e_{j}(k-1)
$$

where $\hat{e}_{j}(k+1 \mid k)$ is the grid voltage extrapolated value.

Returning to the instantaneous phase values $\theta_{j}(k)$, they are used to obtain the sinusoidal references by means of the reference generation block which results in

$$
r_{j}(k)=A(k) \sin \left(\theta_{j}(k)+\phi(k)\right)
$$

where $A(k)$ is the reference amplitude and $\phi(k)$ is the reference phase shift with respect to the grid instantaneous phase. This way it is possible to control the amount of active and reactive power injected to the grid.

In the case of the ISC measurements, they are passed through an FIR filter with $N=3$, obtaining the digitally filtered samples $i_{I j}(k)$ used to compute its error with respect to the reference, which is finally used by the GPC block for each phase. After the addition of the FF correction term, the resulting signal is divided by $v_{\text {bus }} / 2$ which is then fed to the PWM whose switching signals are sent to each of the VSI drivers.

The GPC block contains the TF (36) which has integral action, so an additional anti wind-up (AWU) algorithm as shown in Fig. 8 was added. The error signal $\varepsilon_{j}(k)$ is fed to the numerator block $B\left(z^{-1}\right)$ and its output is added to the output of the denominator block $A\left(z^{-1}\right)$. But the input to this last block is the output of a saturation block (the AWU block) which limits the control signal to the dc voltage levels of the VSI bus. This updates the controller state variables with the 


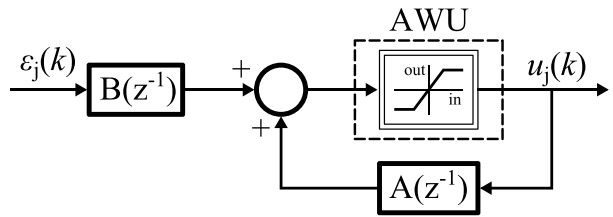

Fig. 8. AWU scheme.

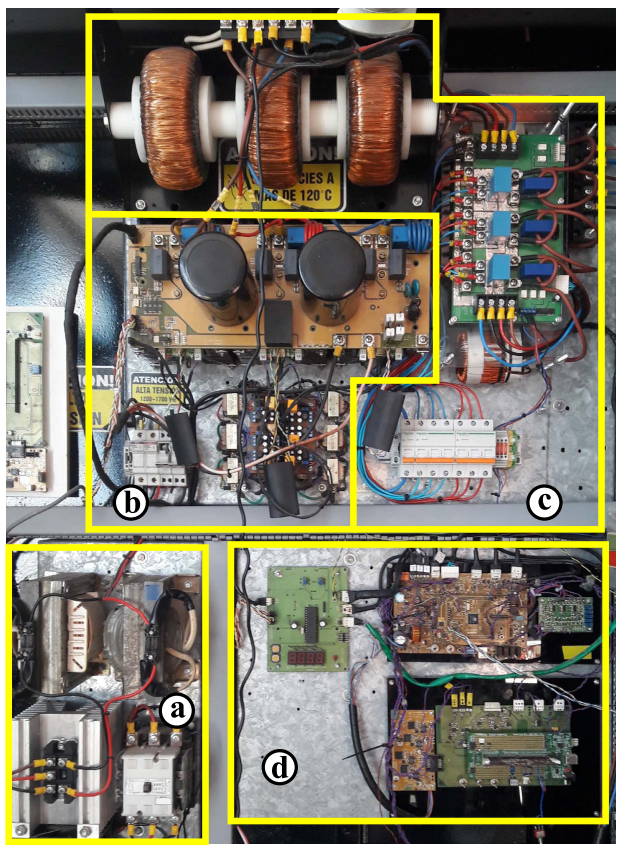

Fig. 9. VSI experimental prototype. (a) Input generator rectifier. (b) VSI and DC-Bus. (c) LCL Filter. (d) Controller boards.

actual control output and, therefore, guarantees the closed-loop operation of the control system at all times.

\section{EXPERIMENTAL RESULTS}

The proposed strategy was tested in an experimental prototype of a 3P3W VSI, as shown in Fig. 9, which was built around Semikron SKM75GB176D 1700V 45A IGBT modules and its dc-bus was built with two $2200 \mu \mathrm{F} 500 \mathrm{~V}$ aluminum capacitors. ISC is sensed with LEM LA-125P Hall-effect current transducers, conditioned, and later sampled by the microcontroller. The digital control framework chosen is composed of a custom board based on TI TMS320F28335 floatingpoint digital signal controller which has an embedded digital signal processor among several useful peripherals including a PWM module. The algorithm calculation time on this platform was $15 \mu$ s long. The LCL components as shown in Fig. 1 are film-type EPCOS (TDK) capacitors B32794D2156K and the damping resistors are Stackpole EWT25JB1R00. Filter inductors were custom made using iron powder cores. Operating conditions and filter component values are as specified in Tables I and II, respectively.

The proposed GPC strategy was compared against a PDB controller, which is the preferred choice in the natural reference frame [26]. It was designed with a low-frequency model assuming an RL load using $L_{L F}=L_{I j}+L_{G j}$ and an
$R_{\text {loss }}$ gathering resistive losses [27]. Considering an additional calculation delay, the resulting PDB controller is

$$
G_{\mathrm{db}}\left(z^{-1}\right)=\frac{22.35-21.65 z^{-1}}{1-z^{-2}}
$$

Nevertheless, the main drawback of this control strategy is its great sensitivity to model mismatch becoming unstable for a modeled inverter side inductance approximately $40 \%$ higher than the real inductance [27]. Therefore, the aim of this comparison is to show that the proposed GPC strategy has similar steady state and transient performance than a PDB controller, but higher robustness against model mismatch.

The steady state behavior of the proposed strategy was evaluated and compared against that of the PDB, and their grid side current waveforms are shown in Fig. 10(a) and (d), respectively. Both controllers were tested connected to a real grid with $\mathrm{THD}_{v}=4.8 \%$ almost at the standard limits. The GPC strategy presents zero steady-state error, in contrast with the PDB controller which due to its lack of integral action, presents a noticeable drop from the desired set point. Although both current controllers present waveform distortion, they meet the IEC61000-3-2 standard but the GPC strategy renders a $\mathrm{THD}_{i}=3.91 \%$ which is less than the $\mathrm{THD}_{i}=4.25 \%$ obtained with the PDB controller.

Transient response was evaluated by means of step changes in current amplitude for both controllers as shown in Fig. 10(b) for the GPC and Fig. 10(e) for the PDB controller, where one of the phases is shown. A step change from $7 \mathrm{~A}_{p}$ to $14 A_{p}$ was set for both controllers, keeping a zero reactive power reference. Both controllers respond rapidly but the PDB presents some ringing inmediately after the step. Also, a full active to reactive power step was evaluated in both strategies as shown in Fig. 12(a) for the GPC and Fig. 12(b) for the PDB controller. Again, as in the case of an active power step, the PDB controller presents some ringing with which last less than $4 \mathrm{~ms}$. In contrast, the proposed GPC strategy presents a transient effect of much shorter duration. The active power magnitude is affected in both cases because grid side current is not the controlled variable.

\section{A. Robustness Tests}

Two robustness tests were carried out for both GPC and PDB controllers, changing the grid-side inductance in one case and the inverter-side inductance in the other, by placing a $1.5 \mathrm{mH}$ inductor in parallel with the original inductor, both in phase $a$ only, with the objective of verifying the robustness criterion used for the GPC design procedure. The resulting inductance variations were $\Delta L_{G a}=-48 \%$ and $\Delta L_{I a}=-53 \%$. In addition, for the inverter-side inductance variation, the GPC stability limits were also proven.

1) Grid-Side Inductance Variation: Reactive power steps were commanded, as shown in Fig. 11. As can be seen, ringing is more pronounced in the PDB strategy than without variations. However, in the GPC strategy, the transient results are similar than in nominal conditions as it was expected by the robustness measures taken in the design process. 


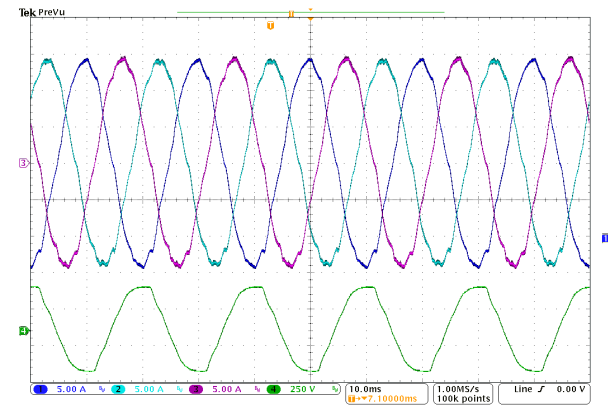

(a)

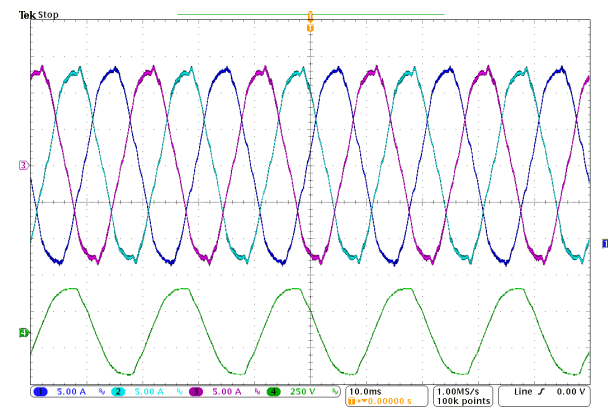

(d)

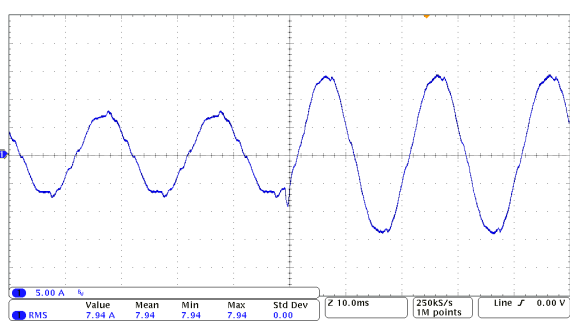

(b)

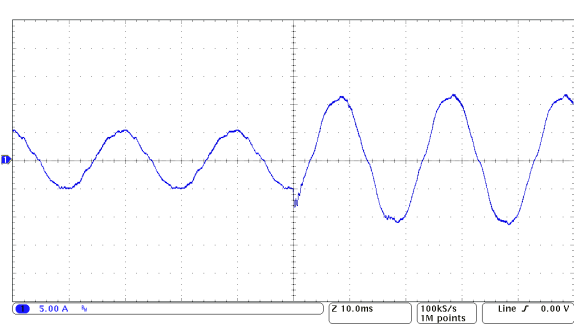

(e)

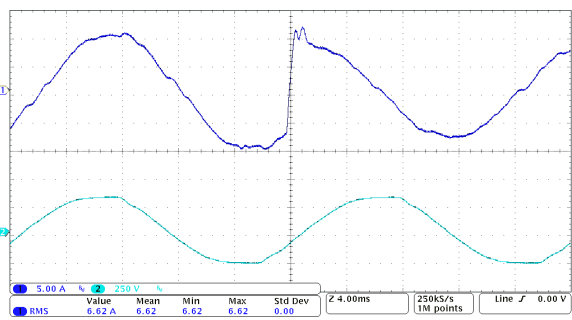

(c)

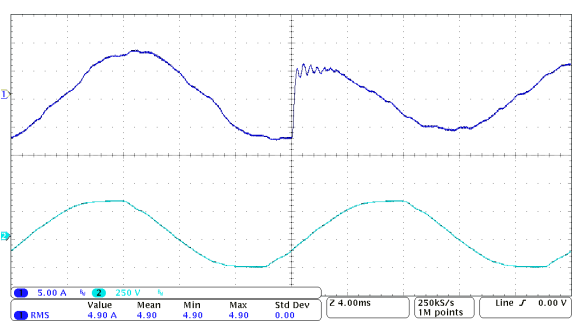

(f)

Fig. 10. (a) and (d) GSC (top) injected to a distorted grid (bottom). $V_{\text {grid }}=218 \mathrm{~V}_{\mathrm{rms}}, \mathrm{THD}_{v}=4.8 \%, I=10 \mathrm{~A}_{\mathrm{rms}}$. (a) $\mathrm{GPC}_{\mathrm{T}} \mathrm{THD}_{i}=3.91 \%$ (d) PDB. $\mathrm{THD}_{i}=4.25 \%$. (b) and (e) GSC step response from $7 \mathrm{Ap}_{\mathrm{p}}$ to $14 \mathrm{~A}_{\mathrm{p}}$. (b) GPC. (e) PDB. (c) and (f) GSC full active to reactive power step response (top) injected to a distorted grid (bottom). (c) GPC. (f) PDB.

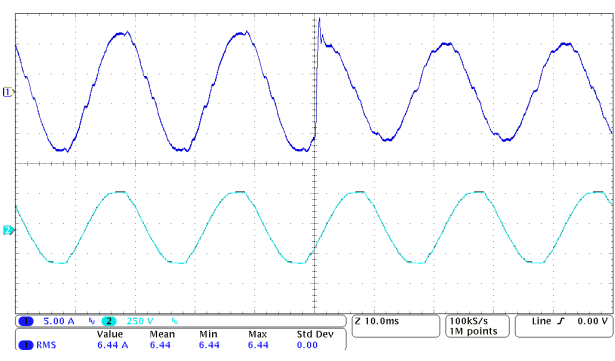

(a)

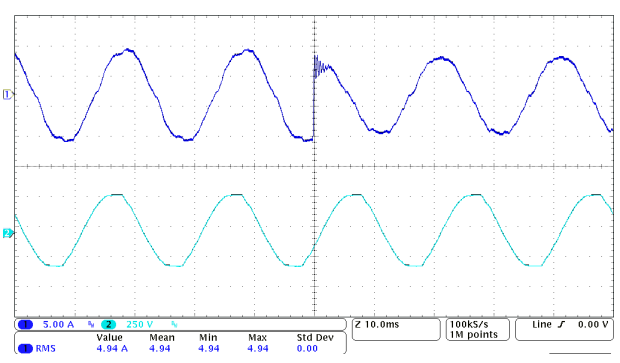

(b)

Fig. 11. GSC full active to reactive power step response (top) injected to a distorted grid (bottom) under grid-side inductance variation. (a) GPC. (b) PDB.

2) Inverter-Side Inductance Variation: As in the previous case, reactive power steps were commanded as shown in Fig. 12. In this case, model mismatch results in sustained oscillations for almost a half-cycle in the PDB strategy, showing a lower stability margin than with the nominal values. However, in the GPC strategy, the transient results do not

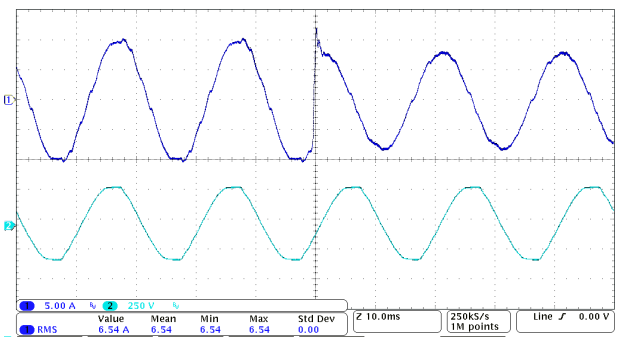

(a)

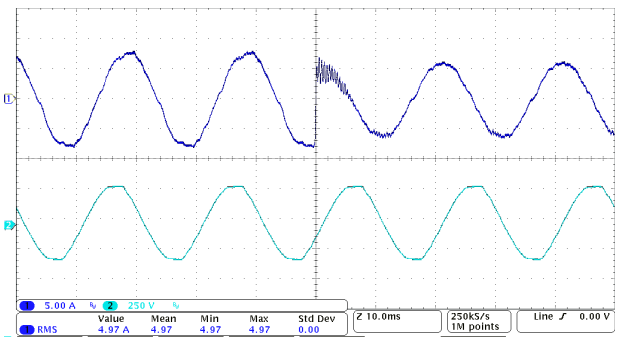

(b)

Fig. 12. GSC full active to reactive power step response (top) injected to a distorted grid (bottom) under inverter-side inductance variation. (a) GPC. (b) PDB.

show any kind of oscillation which it also was expected by the robustness measures taken in the design process, as the inductance variation was inside the stability region for the chosen $\lambda$ value as previously shown.

3) Stability Limits: With $\Delta L_{I a}=-53 \%$, the GPC $\lambda$ value was changed from $\lambda=0.15$ to $\lambda=0.08$. This change resulted 


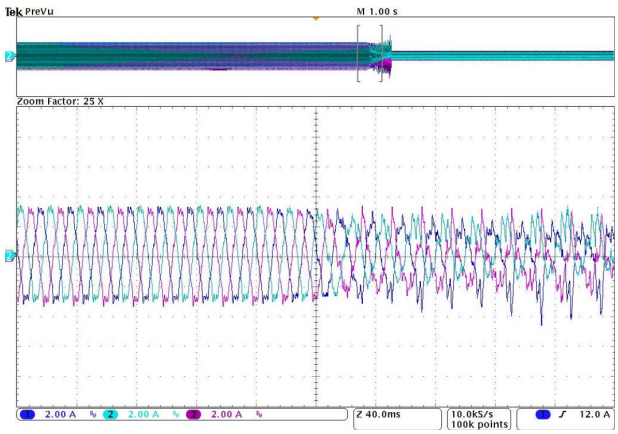

Fig. 13. GSCs obtained with GPC transitioning from the stable $(\lambda=0.15)$ to the unstable $(\lambda=0.08)$ region.

in the GPC transitioning from the stable region to the unstable region, with the resulting GSCs shown in Fig. 13. The distorted waveform is due to the oscillation of the control input in the unstable region. This result shows that control robustness can be impaired by the improper selection of the $\lambda$ value.

\section{CONCLUSION}

An ISC control strategy based on GPC subtype of CCS-MPC was proposed for LCL grid-connected VSIs. Even though the GPC strategy was used before with first-order filter models, for its implementation in LCL grid-connected VSIs, it was shown that an LCL full-order model gives better stability margins than using a first-order model, which might also be the case when using other MPC strategies. Then, a sensitivity analysis was carried out obtaining the most critical component variations affecting LCL admittance up to the GPC maximum bandwidth, which were both the inverter-side and the grid-side inductors. Based on these results, a maximum allowed variation of inductance was calculated as a function of GPC cost-function parameter $\lambda$, where the inverter-side inductor resulted the most critical component for GPC stability. Following this analysis, the optimal $\lambda$ value was chosen maximizing robustness while meeting current quality standards even under a distorted grid voltage.

Experimental results showed that the proposed GPC strategy met current quality standards and performed similarly under different LCL component variations: specifically both under grid-side and inverter-side inductance variations up to approximately half of their nominal value. Its performance was superior than a PDB controller, having a comparable response speed but better robustness against LCL components variations, especially under inverter-side inductance reduction. Also, the GPC stability limits were experimentally proven. Finally, this paper provides proof that an MPC strategy can be used in grid-connected VSIs with LCL filters without the need for any model-based active damping strategy and, by using a GPC subtype, speed and robustness against model mismatch can be achieved while keeping computational burden low, allowing its implementation in standard industrial controllers.

\section{REFERENCES}

[1] J. M. Carrasco et al., "Power-electronic systems for the grid integration of renewable energy sources: A survey," IEEE Trans. Ind. Electron., vol. 53, no. 4, pp. 1002-1016, Jun. 2006, doi: 10.1109/TIE.2006.878356.
[2] A. Aapro, T. Messo, T. Roinila, and T. Suntio, "Effect of active damping on output impedance of three-phase grid-connected converter," IEEE Trans. Ind. Electron., vol. 64, no. 9, pp. 7532-7541, Sep. 2017, doi: 10. 1109/TIE.2017.2696494.

[3] D. Pan, X. Ruan, X. Wang, H. Yu, and Z. Xing, "Analysis and design of current control schemes for LCL-type grid-connected inverter based on a general mathematical model," IEEE Trans. Power Electron., vol. 32, no. 6, pp. 4395-4410, Jun. 2017, doi: 10.1109/TPEL.2016.2602219.

[4] R. Guzman, L. G. de Vicuña, J. Morales, M. Castilla, and J. Miret, "Model-based active damping control for three-phase voltage source inverters with LCL filter," IEEE Trans. Power Electron., vol. 32, no. 7, pp. 5637-5650, Jul. 2017, doi: 10.1109/TPEL.2016.2605858.

[5] N. Panten, N. Hoffmann, and F. W. Fuchs, "Finite control set model predictive current control for grid-connected voltage-source converters with LCL filters: A study based on different state feedbacks," IEEE Trans. Power Electron., vol. 31, no. 7, pp. 5189-5200, Jul. 2016, doi: 10. 1109/TPEL.2015.2478862.

[6] Y. He, H. S.-H. Chung, C. N.-M. Ho, and W. Wu, "Use of boundary control with second-order switching surface to reduce the system order for deadbeat controller in grid-connected inverter," IEEE Trans. Power Electron., vol. 31, no. 3, pp. 2638-2653, Mar. 2016, doi: 10. 1109/TPEL.2015.2441117.

[7] P. Cortés, J. Rodríguez, D. E. Quevedo, and C. Silva, "Predictive current control strategy with imposed load current spectrum," IEEE Trans. Power Electron., vol. 23, no. 2, pp. 612-618, Mar. 2008, doi: 10. 1109/TPEL.2007.915605.

[8] M. G. Judewicz, J. R. Fischer, N. Echeverría, S. A. González, and D. O. Carrica, "Filtro notch digital para la conformación del espectro en control predictivo basado en modelo," in Proc. IEEE Biennial Congr. Argentina (ARGENCON), Jun. 2014, pp. 1-6, doi: 10.1109/ARGENCON.2014.6868464.

[9] X. Fu and S. Li, "Control of single-phase grid-connected converters with LCL filters using recurrent neural network and conventional control methods," IEEE Trans. Power Electron., vol. 31, no. 7, pp. 5354-5364, Jul. 2016, doi: 10.1109/TPEL.2015.2490200.

[10] C. Citro, P. Siano, and C. Cecati, 'Designing inverters' current controllers with resonance frequencies cancellation," IEEE Trans. Ind. Electron., vol. 63, no. 5, pp. 3072-3080, May 2016, doi: 10.1109/TIE. 2016.2516507.

[11] J. Wang, J. D. Yan, and L. Jiang, "Pseudo-derivative-feedback current control for three-phase grid-connected inverters with LCL filters," IEEE Trans. Power Electron., vol. 31, no. 5, pp. 3898-3912, May 2016, doi: 10.1109/TPEL.2015.2462331.

[12] M. P. S. Gryning, Q. Wu, M. Blanke, H. H. Niemann, and K. P. H. Andersen, "Wind turbine inverter robust loop-shaping control subject to grid interaction effects," IEEE Trans. Sustain. Energy, vol. 7, no. 1, pp. 41-50, Jan. 2016, doi: 10.1109/TSTE.2015.2472285.

[13] C. A. Busada, S. G. Jorge, and J. A. Solsona, "Full-state feedback equivalent controller for active damping in $L C L$-filtered gridconnected inverters using a reduced number of sensors," IEEE Trans. Ind. Electron., vol. 62, no. 10, pp. 5993-6002, Oct. 2015, doi: 10 1109/TIE.2015.2424391.

[14] J. Castelló, J. M. Espí, and R. García-Gil, "A new generalized robust predictive current control for grid-connected inverters compensates antialiasing filters delay," IEEE Trans. Ind. Electron., vol. 63, no. 7, pp. 4485-4494, Jul. 2016, doi: 10.1109/TIE.2015.2497303.

[15] S. Mariethoz and M. Morari, "Explicit model-predictive control of a PWM inverter with an LCL filter," IEEE Trans. Ind. Electron., vol. 56, no. 2, pp. 389-399, Feb. 2009, doi: 10.1109/TIE.2008.2008793.

[16] J. R. Fischer, S. A. Gonzalez, M. A. Herran, M. G. Judewicz, and D. O. Carrica, "Calculation-delay tolerant predictive current controller for three-phase inverters," IEEE Trans. Ind. Informat., vol. 10, no. 1, pp. 233-242, Feb. 2014, doi: 10.1109/TII.2013.2276104.

[17] M. G. Judewicz, S. A. González, N. I. Echeverría, J. Fischer, and D. Carrica, "Generalized predictive current control (GPCC) for gridtie three-phase inverters," IEEE Trans. Ind. Electron., vol. 63, no. 7, pp. 4475-4484, Jul. 2016, doi: 10.1109/TIE.2015.2508934.

[18] K. J. Astrom and B. Wittenmark, Computer-Controlled Systems, 3rd ed. Upper Saddle River, NJ, USA: Prentice-Hall, 1997.

[19] D. Clarke, Advances in Model-Based Predictive Control. New York, NY, USA: Oxford Univ. Press, 1994.

[20] K. Ogata, Discrete-Time Control Systems. Englewood Cliffs, NJ, USA: Prentice-Hall, 1995.

[21] D. W. Clarke and C. Mohtadi, "Properties of generalized predictive control," Automatica, vol. 25, no. 6, pp. 859-875, 1989, doi: https://doi. org/10.1016/0005-1098(89)90053-8 
[22] M. A. Herrán, J. R. Fischer, S. A. González, M. G. Judewicz, and D. O. Carrica, "Adaptive dead-time compensation for grid-connected PWM inverters of single-stage PV systems," IEEE Trans. Power Electron., vol. 28, no. 6, pp. 2816-2825, Jun. 2013, doi: 10.1109/TPEL.2012. 2227811.

[23] A. Kouchaki, M. Nymand, and R. Lazar, "Non-iterative, analyticbased passive lcl filter design approach for three-phase two-level power factor correction converters," in Proc. 18th Eur. Conf. Power Electron. Appl. (EPE ECCE Europe), Sep. 2016, pp. 1-10, doi: 10.1109/ EPE.2016.7695424.

[24] I. Carugati, S. Maestri, P. G. Donato, D. Carrica, and M. Benedetti, "Variable sampling period filter pll for distorted three-phase systems," IEEE Trans. Power Electron., vol. 27, no. 1, pp. 321-330, Jan. 2012, doi: 10.1109/TPEL.2011.2149542.

[25] J. C. Moreno, J. M. E. Huerta, R. G. Gil, and S. A. González, "A robust predictive current control for three-phase grid-connected inverters," IEEE Trans. Ind. Electron., vol. 56, no. 6, pp. 1993-2004, Jun. 2009.

[26] F. Blaabjerg, R. Teodorescu, M. Liserre, and A. V. Timbus, "Overview of control and grid synchronization for distributed power generation systems," IEEE Trans. Ind. Electron., vol. 53, no. 5, pp. 1398-1409, Oct. 2006, doi: 10.1109/TIE.2006.881997.

[27] Y. A.-R. I. Mohamed, M. A. Rahman, and R. Seethapathy, "Robust line-voltage sensorless control and synchronization of LCL-filtered distributed generation inverters for high power quality grid connection," IEEE Trans. Power Electron., vol. 27, no. 1, pp. 87-98, Jan. 2012, doi: 10.1109/TPEL.2011.2159866.

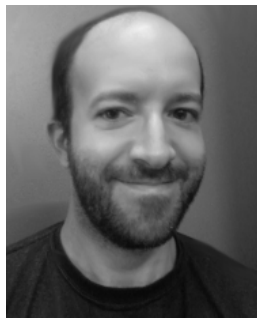

Marcos Gabriel Judewicz (S'11-M'15) was born in Mar del Plata, Argentina, in 1986. He received the Ing. and Ph.D. degrees in electronic engineering from the Universidad Nacional de Mar del Plata (UNMdP), Mar del Plata, in 2011 and 2016, respectively.

In 2016, he joined the Department of Electronic Engineering, UNMdP, as a Graduate Assistant. He is currently an Assistant Researcher with the Instituto de Investigaciones Científicas y Tecnológicas en Electrónica, UNMdP, and also with the Consejo Nacional de Investigaciones Científicas y Técnicas, Buenos Aires, Argentina. His current research interests include power electronics and predictive control. Dr. Judewicz is member of the IEEE Industrial Electronics Society.

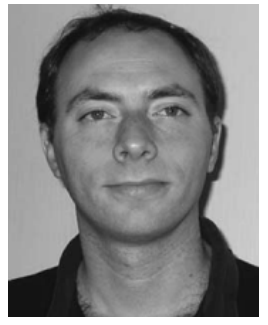

Sergio Alejandro González (M'01) was born in Mar del Plata, Argentina, in 1972. He received the Ing. and Dr.-Ing. degrees in electronic engineering from the Universidad Nacional de Mar del Plata (UNMdP), Mar del Plata, in 1999 and 2006, respectively.

Since 1999, he has been an Assistant Professor of control systems with the School of Engineering, UNMdP. He is currently a Researcher with the Instituto de Investigaciones Científicas y Tecnológicas en Electrónica, UNMdP, and a member of the National Scientific and Technical Research Council, Buenos Aires, Argentina. His current research interests include hardware designs, digital signal processing, digital control techniques for electrical power systems, and integration of distributed energy systems.

Dr. González is a member of the IEEE Industrial Electronics Society.

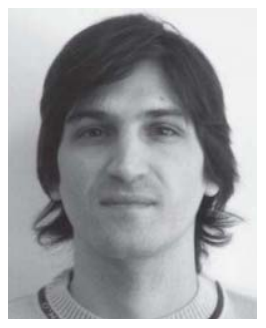

Jonatan Roberto Fischer (S'09-M'14) received the Ing. and Dr.-Ing. degrees in electronics from the Universidad Nacional de Mar del Plata (UNMdP), Mar del Plata, Argentina, in 2008 and 2013, respectively.

He is currently a Teaching Assistant of control systems with the School of Engineering, UNMdP. His current research interests include power electronics, control systems, and digital signal processing.

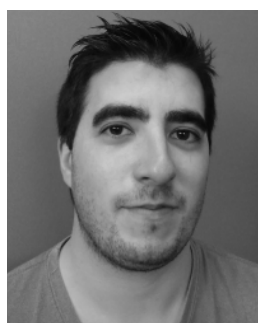

Juan Francisco Martínez was born in Mar del Plata, Argentina in 1989. He received the Ing. degree in electromechanical engineering from the Universidad Nacional de Mar del Plata (UNMdP), Mar del Plata, in 2017, where is currently pursuing the Ph.D. degree in electronic engineering.

His current research interests include wind power systems and predictive control.

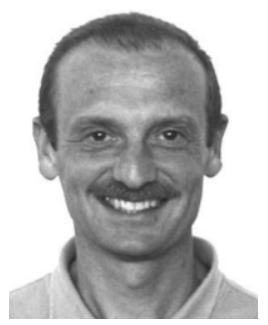

Daniel Oscar Carrica (S'85-M'95-SM'08) was born in Dolores, Argentina, in 1958. He received the B.S. degree in engineering from the Universidad Nacional de Mar del Plata (UNMdP), Mar del Plata, Argentina, in 1984, the M.Sc. degree in electronics from the Universidad Politécnica de Madrid, Madrid, Spain, in 1992, and the Dr.-Ing. degree in electronic engineering from UNMdP, in 2006.

In 1984, he joined the Department of Electronics, UNMdP, where he is currently a Full Professor. From 1990 to 1992, he was an Associate Scientist at the European Organization for Nuclear Research (CERN), Geneva, Switzerland. He is currently also the Head of the Facultad de Ingeniería, Instituto de Investigaciones Científicas y Tecnológicas en Electrónica, UNMdP. 